\title{
Preface to RIGiM 2008
}

\author{
Colette Rolland ${ }^{1}$, Carson $\mathrm{Woo}^{2}$, and Camille Salinesi ${ }^{1}$ \\ ${ }^{1}$ Université Paris 1 Panthéon - Sorbonne, France \\ ${ }^{2}$ University of British Columbia, Canada
}

The use of intentional concepts, the notion of "goal" in particular, has been prominent in recent approaches to requirement engineering (RE). Goal-oriented frameworks and methods for requirements engineering (GORE) have been keynote topics in requirements engineering, conceptual modelling, and more generally in software engineering. What are the conceptual modelling foundations in these approaches? RIGiM (Requirements Intentions and Goals in Conceptual Modelling) aims to provide a forum for discussing the interplay between requirements engineering and conceptual modelling, and in particular, to investigate how goal- and intention-driven approaches help in conceptualising purposeful systems. What are the fundamental objectives and premises of requirements engineering and conceptual modelling respectively, and how can they complement each other? What are the demands on conceptual modelling from the standpoint of requirements engineering? What conceptual modelling techniques can be further taken advantage of in requirements engineering? What are the upcoming modelling challenges and issues in GORE? What are the unresolved open questions? What lessons are there to be learnt from industrial experiences? What empirical data are there to support the cost-benefit analysis when adopting GORE methods? Are there applications domains or types of project settings for which goals and intentional approaches are particularly suitable or not suitable? What degree of formalization and automation or interactivity are feasible and appropriate for what types of participants during requirements engineering? e.g., business domain stakeholders, requirements modellers, ontology engineers, etc. 